Este documento es la versión enviada el 16/09/2018 para su evaluación por la organización del congreso. EL ENVÍO DE LA PONENCIA SUPONE AUTOMÁTICAMENTE LA CESIÓN DE DERECHOS DE PUBLICACIÓN A 'FÓRUM INTERNACIONAL DE COMUNICACIÓN Y RELACIONES PÚBLICAS' (FÓRUM XXI)

CONGRESO UNIVERSITARIO INTERNACIONAL SOBRE LA COMUNICACIÓN EN LA PROFESIÓN Y EN LA UNIVERSIDAD DE HOY: CONTENIDOS, INVESTIGACIÓN, INNOVACIÓN Y DOCENCIA (CUICIID 2018 Virtual y En Línea) 


\title{
Relación entre el uso de las bibliotecas universitarias y el desarrollo académico: el caso de las universidades públicas españolas y los estudiantes de grado
}

\author{
Pedro Lázaro-Rodríguez ${ }^{1}$
}

Pedro Lázaro Rodríguez es beneficiario de una de las ayudas para contratos predoctorales de Formación de Profesorado Universitario del Ministerio de Educación, Cultura y Deporte de España (Referencia FPU-2014/04213). El trabajo está soportado por el Ministerio de Economía y Competitividad de España (Referencia TIN2016-75850-R).

Las bibliotecas son uno de los servicios mejor valorados por la sociedad. En el marco concreto de la universidad como institución, las bibliotecas universitarias pueden servir como recurso de apoyo a la investigación, formación y aprendizaje de los estudiantes. En este trabajo se analiza la relación entre el uso de las bibliotecas de las universidades públicas españolas y una serie de indicadores relacionados con el desarrollo académico. Para ello, se lleva a cabo un análisis estadístico basado en el cálculo de los coeficientes de correlación lineal de Pearson y Spearman para estudiar la existencia o no de significación estadística y la relación entre las variables. Como resultados, destaca la existencia de significación estadística entre las variables de préstamos en las bibliotecas y las tasas de rendimiento y éxito académico, eficiencia, graduación, y duración media en los estudios de estudiantes de grado, siendo la correlación positiva en los 4 primeros casos y negativa en el último. Por tanto, se concluye, sin atribuir relación causal, que en la muestra estudiada sucede que donde mayores son los préstamos en las bibliotecas universitarias, mayores son también el rendimiento y éxito académicos, la eficiencia y la tasa de graduación de los estudiantes de grado, y menor es la duración media de dichos

1

Pedro Lázaro Rodríguez es contratado predoctoral FPU en el Departamento de Información y Comunicación de la Universidad de Granada (España). 
estudiantes en los respectivos estudios. Con todo, esta investigación sirve como aporte de valor a las bibliotecas universitarias en el marco de las instituciones a las que pertenecen, pues un mayor uso de las mismas puede reportar beneficios a los estudiantes en el sentido de su desarrollo académico.

\section{Introducción}

Las bibliotecas destacan por ser uno de los servicios mejor valorados por la sociedad en la que se hallan y por la comunidad que hace uso y disfruta de sus servicios (Kwak y Yoo, 2012, p. 263). Tal es así, que se las considera como tercer lugar junto al hogar y al trabajo (Houghton, Foth y Miller, 2013, p. 28), siendo defendidas por los beneficios que aportan a la comunidad en el contexto actual de la sociedad de la información (Herrera-Viedma y López-Gijón, 2013, p. 1382).

Aun considerando la buena imagen que por lo general se tiene de las bibliotecas, los esfuerzos por mejorarlas no deben cesar en un ejercicio de continua evaluación de sus servicios. Ejemplos concretos de estos esfuerzos se encuentran en la literatura científica más actual, por ejemplo, en un estudio que trata de la modelización del uso de las bibliotecas públicas españolas en un estudio comparativo con las de Finlandia (Lázaro-Rodríguez y Vakkari, 2018, p. 1); en otro trabajo que analiza la visibilidad de las bibliotecas públicas españolas en medios de comunicación españoles proponiendo una serie de medidas para su mayor uso y promoción (Lázaro-Rodríguez, López-Gijón y Herrera-Viedma, 2018, p. 1); o con el desarrollo e implementación de la herramienta digital abierta Secaba-Rank para la evaluación de bibliotecas públicas y universitarias españolas (Lázaro-Rodríguez, López-Gijón, Alonso, Martínez-Sánchez, y Herrera-Viedma, 2018, p. 279).

Al respecto de las bibliotecas universitarias, pueden concebirse como entidades que precisan demostrar el valor de sus servicios para justificar su existencia dentro de las universidades, entendidas estas como instituciones de las que las bibliotecas universitarias forman parte (Bustos-González, 2007, p. 281). Desde ahí, como aporte de valor a las bibliotecas universitarias, pueden entenderse los objetivos y la pregunta de investigación que dan pié a este estudio.

El objetivo de este trabajo es aportar valor a las bibliotecas universitarias públicas españolas analizando la relación desde un estudio estadístico entre el uso de dichas bibliotecas, entendido desde los préstamos y las visitas, con una serie de variables relacionadas todas con el desarrollo o éxito académico de estudiantes de grado, a saber, las tasas de éxito académico, rendimiento académico, eficiencia, abandono, graduación, y la tasa de duración media en los estudios. Con todo, la pregunta de investigación que se desea resolver puede ser planteada de la siguiente forma: ¿existe relación entre el uso de las bibliotecas 
universitarias públicas españolas y el desarrollo académico de estudiantes de grado? En ese sentido, la cuestión sobre qué tipo de relación se da entre ambos aspectos se puede considerar como implícita en la anterior.

Para todo ello, el trabajo se estructura en lo que sigue con una sección para una revisión de la literatura acorde al tema del presente trabajo seguida de la explicación de la metodología y materiales empleados. Después de ello, se presentan los resultados y su interpretación. Por último, se añade una sección para la discusión de lo alcanzado y una conclusión sobre todo lo llevado a cabo como cierre del trabajo.

\section{Revisión de la literatura}

En la literatura científica encontramos diversos estudios de naturaleza estadística con el objetivo de aportar valor a las bibliotecas universitarias justificando su existencia y legitimando su labor. En el ámbito internacional, existen estudios que analizan la relación entre la colección de revistas electrónicas con su uso, y la publicación y el impacto de los trabajos de los investigadores en universidades francesas (Boukacem-Zeghmouri, Bador, Lafouge y Prost, 2016, p. 263), o entre el uso de las bibliotecas con la retención de estudiantes en la universidad (Murray, Ireland, y Hackathorn, 2016, p. 631). En un sentido similar, existen trabajos que correlacionan la inversión en bibliotecas con la excelencia académica en universidades del Reino Unido (Oppenheim y Stuart, 2004, p. 156), o variables de la estructura de las páginas web en bibliotecas académicas y sus características en los Estados Unidos de América (Orduña-Malea y Regazzi, 2014, p. 315). A su vez, también destacan los estudios que analizan la relación entre el uso de las bibliotecas con variables del entorno socioeconómico en estudiantes de primer año (Soria, Nackerud y Peterson, 2015, p. 636).

Delimitando la revisión al caso concreto de la relación entre bibliotecas universitarias y éxito académico, se pueden mencionar estudios llevados a cabo para universidades en países como Kuwait (Alharbi y Middleton, 2011, p. 86), Brasil (Freitas-Hübner y Silveira-Andretta, 2016, p. 45), Sudáfrica (De Jager, Nassimbeni, Daniels y D’Angelo, 2017, p. 40), Ghana (Owusu-Acheaw, 2014, p. 1), Japón (Wong y Webb, 2011, p. 361), o Croacia (Morić-Filipović, 2012, p. 65). A su vez, destacan estudios generales y que no se limitan a una sola universidad de un único país (Powell, 1992, p. 245; Yoon y Young, 2008, p. 345; Chohan, Bhatti, y Naeem, 2018, p. 557).

Por último y al nivel nacional de España, destacan estudios de base estadística analizando la relación entre el presupuesto de las bibliotecas y la productividad investigadora (Fernández y Rubio, 2013, p. 1), y otros más recientes acerca de la contribución de las bibliotecas universitarias a la consecución de los proyectos y planes de las instituciones a las que pertenecen 
(Alonso-Arévalo y Vázquez-Vázquez, 2018, p. 42).

\section{Metodología y materiales}

El presente estudio consiste en el análisis de la relación entre el uso de las bibliotecas públicas universitarias españolas con indicadores relacionados con el desarrollo académico en estudiantes de grado. En concreto y en referencia a los materiales empleados, para lo primero se utilizan los indicadores de préstamos y visitas per cápita en el año 2015 y para lo segundo las tasas de éxito académico, rendimiento académico, eficiencia, abandono, graduación, y la de duración media en los estudios referidas al curso 2015-2016.

Los datos para la información sobre bibliotecas se han obtenido de la Red de Bibliotecas Universitarias Españolas (Rebiun, 2018). Los préstamos per cápita consisten en la suma de los préstamos domiciliarios y los documentos descargados de los recursos electrónicos del apartado datos counter, dividido entre la población, que consiste en la suma de estudiantes (de grado, posgrado y titulaciones propias) y docentes (con dedicación a tiempo completo y parcial). Las visitas per cápita consisten en las entradas a la biblioteca divididas entre la población. Cabe decir que se ha considerado como población a la anterior y no solo a los estudiantes de grado porque en los datos de Rebiun no se distinguen los préstamos ni las visitas por tipo de usuario o población. Con todo, las correlaciones calculadas pueden considerarse válidas por representar los estudiantes de grado la mayor parte del porcentaje de la población total. A su vez, se han considerado las bibliotecas y universidades públicas con datos disponibles para las variables incluidas en el estudio, eliminando los casos en que faltaba alguno de los datos requeridos y resultando 41 válidas en total.

Por su lado, la información referida al desarrollo académico se ha obtenido del Anuario de indicadores universitarios 2017 del Ministerio de Educación, Cultura y Deporte (MECD, 2018). De acuerdo a la fuente, La tasa de rendimiento consiste en la relación porcentual entre el número de créditos superados por los estudiantes matriculados en un curso académico (excluyendo créditos reconocidos y transferidos) y el número total de créditos matriculados en dicho curso académico; la tasa de éxito, en la relación porcentual entre el número de créditos superados por los estudiantes matriculados en un curso académico (excluyendo créditos reconocidos y transferidos) y el número total de créditos presentados a examen en dicho curso académico; la tasa de eficiencia, en la relación porcentual entre el número de créditos superados a lo largo de la titulación por los estudiantes egresados (excluyendo créditos reconocidos y transferidos) y el número total de créditos matriculados; la tasa de abandono del estudio, en el porcentaje de estudiantes de una cohorte de nuevo ingreso en el curso 2011-2012, matriculados en alguno de los títulos de las universidades, que sin haberse graduado en ese título no se han matriculado 
en él durante dos cursos seguidos; la duración media de los estudios, en el número medio de años que tardan los estudiantes en graduarse; y la tasa de graduación, en el porcentaje de estudiantes que finalizan la titulación en el tiempo teórico previsto o en un curso más.

En cuanto a la metodología, la relación entre el uso de las bibliotecas y la información referida al desarrollo académico se lleva a cabo en base a un análisis de la correlación lineal. Para ello, en primer lugar se ha estudiado la normalidad de las variables mediante el test de Shapiro Wilk, obteniendo que todas menos la duración media de los estudios siguen una distribución normal. Por ello, para la relación entre dicha variable y los préstamos y las visitas a las bibliotecas se ha utilizado el coeficiente de Spearman, mientras que para el resto de correlaciones se ha utilizado el coeficiente de Pearson. El análisis estadístico sigue el método de otros estudios con base estadística sobre bibliotecas ya mencionados en el presente trabajo (Fernández y Rubio, 2013; LázaroRodríguez, López-Gijón y Herrera-Viedma, 2018; Lázaro-Rodríguez y Vakkari, 2018).

Por último, cabe especificar que para la unificación y procesamiento de los datos se ha utilizado el software LibreOffice Calc 6.0.5.5. Por su lado, para todo lo relacionado al análisis estadístico se ha utilizado el software BM SPSS 24.0 Desktop Linux Client Multilingual.

\section{Resultados}

En primer lugar y antes de presentar los resultados de las correlaciones, la información para los indicadores calculados a partir de los datos especificados del uso de las bibliotecas sobre los préstamos per cápita (PPC) y visitas per cápita (VPC), y la información referida al desarrollo académico sobre la tasa de rendimiento (TR), tasa de éxito (TEX), tasa de eficiencia (TEF), tasa global de abandono cohorte 2011-2012 (TGA), duración media de los estudios (DM) y la tasa de graduación (TG), se pueden ver en la Tabla 1.

\begin{tabular}{|l|c|c|c|c|c|c|c|c|}
\hline \multicolumn{1}{|c|}{ Biblioteca/Universidad } & PPC & VPC & TR & TEX & TEF & TGA & DM & TG \\
\hline U. de Alcalá & 12.31 & 41.81 & 80.87 & 87.83 & 92.00 & 28.22 & 4.51 & 56.00 \\
\hline U. d'Alacant & 22.91 & 55.98 & 80.26 & 85.64 & 92.59 & 30.50 & 4.58 & 48.00 \\
\hline U. de Almería & 41.73 & 40.19 & 75.48 & 87.19 & 91.86 & 28.57 & 4.49 & 50.10 \\
\hline U. Autònoma de Barcelona & 69.74 & 67.94 & 85.11 & 90.37 & 94.55 & 28.13 & 4.48 & 57.20 \\
\hline U. Autónoma de Madrid & 55.75 & 43.41 & 88.14 & 92.08 & 94.73 & 24.28 & 4.41 & 61.50 \\
\hline U. de Barcelona & 41.27 & 40.98 & 84.20 & 90.53 & 93.08 & 29.30 & 4.60 & 55.50 \\
\hline U. de Cádiz & 43.78 & 49.64 & 77.81 & 86.22 & 92.43 & 34.63 & 4.56 & 40.80 \\
\hline
\end{tabular}




\begin{tabular}{|c|c|c|c|c|c|c|c|c|}
\hline U. de Cantabria & 33.71 & 101.69 & 76.82 & 84.56 & 91.06 & 30.75 & 4.67 & 42.80 \\
\hline U. Carlos III de Madrid & 36.18 & 71.30 & 83.45 & 90.02 & 90.60 & 23.82 & 4.76 & 52.40 \\
\hline U. Castilla-La Mancha & 20.77 & 54.86 & 78.98 & 87.14 & 90.44 & 27.17 & 4.68 & 40.60 \\
\hline U. de Córdoba & 35.83 & 74.21 & 76.87 & 87.86 & 91.50 & 28.63 & 4.49 & 37.40 \\
\hline U. da Coruña & 35.78 & 43.29 & 71.52 & 84.01 & 87.10 & 24.93 & 4.83 & 44.50 \\
\hline U. de Extremadura & 19.20 & 51.33 & 79.19 & 87.01 & 89.11 & 24.93 & 4.90 & 44.60 \\
\hline U. de Girona & 27.37 & 75.65 & 85.20 & 89.94 & 93.86 & 29.58 & 4.51 & 55.20 \\
\hline U. de Granada & 33.12 & 39.03 & 80.27 & 89.94 & 93.54 & 28.26 & 4.52 & 44.20 \\
\hline U. de Jaén & 38.98 & 38.87 & 77.51 & 85.72 & 90.83 & 31.74 & 4.53 & 48.40 \\
\hline U. Jaume I & 29.56 & 53.86 & 77.81 & 87.00 & 91.64 & 32.35 & 4.62 & 42.50 \\
\hline U. de La Laguna & 26.09 & 49.77 & 73.97 & 88.03 & 89.25 & 39.05 & 4.65 & 38.70 \\
\hline U. de La Rioja & 38.90 & 59.19 & 78.24 & 86.17 & 89.33 & 33.77 & 4.54 & 56.50 \\
\hline U. de Lleida & 29.18 & 72.23 & 84.83 & 89.84 & 91.96 & 26.16 & 4.52 & 53.20 \\
\hline U. de Málaga & 33.04 & 74.00 & 75.61 & 87.33 & 92.50 & 30.25 & 4.44 & 38.30 \\
\hline U. Miguel Hernández & 25.11 & 16.52 & 75.40 & 85.14 & 91.70 & 30.14 & 4.54 & 38.10 \\
\hline U. de Murcia & 17.48 & 53.76 & 77.48 & 88.48 & 89.50 & 27.23 & 4.67 & 49.30 \\
\hline U. de Oviedo & 39.60 & 27.01 & 75.03 & 84.91 & 89.67 & 33.72 & 4.67 & 40.30 \\
\hline U. Pablo de Olavide & 40.89 & 42.80 & 82.98 & 90.51 & 92.26 & 22.40 & 4.61 & 35.70 \\
\hline U. del País Vasco & 35.87 & 32.82 & 77.52 & 88.34 & 91.39 & 25.34 & 4.51 & 51.70 \\
\hline U. de Las P. de Gran Canaria & 22.94 & 35.11 & 75.69 & 84.49 & 90.00 & 37.43 & 4.68 & 33.60 \\
\hline U. Politécnica de Cartagena & 19.69 & 76.11 & 62.42 & 80.58 & 85.49 & 37.65 & 5.16 & 24.10 \\
\hline U. Politècnica de Catalunya & 33.09 & 38.97 & 77.02 & 81.11 & 89.35 & 40.03 & 4.90 & 40.90 \\
\hline U. Politécnica de Madrid & 17.31 & 54.00 & 69.90 & 78.29 & 85.86 & 31.11 & 5.23 & 28.50 \\
\hline U. Politècnica de València & 44.77 & 49.45 & 83.32 & 88.61 & 92.27 & 35.38 & 4.73 & 42.70 \\
\hline U. Pompeu Fabra & 77.38 & 122.65 & 89.72 & 93.47 & 95.29 & 32.45 & 4.35 & 66.10 \\
\hline U. Pública de Navarra & 40.08 & 88.12 & 80.42 & 86.82 & 90.31 & 36.53 & 4.46 & 52.70 \\
\hline U. Rey Juan Carlos & 6.89 & 30.22 & 80.48 & 87.81 & 89.53 & 22.49 & 4.92 & 40.80 \\
\hline U. Rovira i Virgili & 72.71 & 46.35 & 83.03 & 89.86 & 93.67 & 27.79 & 4.42 & 58.30 \\
\hline U. de Salamanca & 28.95 & 58.87 & 81.22 & 87.93 & 91.18 & 19.61 & 4.49 & 57.20 \\
\hline U. de Santiago de Compostela & 26.88 & 14.31 & 76.92 & 86.01 & 90.90 & 24.89 & 4.64 & 53.20 \\
\hline U. de Sevilla & 50.38 & 54.32 & 73.00 & 86.23 & 87.56 & 28.06 & 4.82 & 42.20 \\
\hline U. de València & 43.88 & 52.97 & 84.11 & 91.60 & 93.09 & 21.72 & 4.47 & 62.00 \\
\hline U. de Valladolid & 21.33 & 75.33 & 79.49 & 86.91 & 91.19 & 22.29 & 4.58 & 53.60 \\
\hline U. de Zaragoza & 24.65 & 68.03 & 79.18 & 87.54 & 91.78 & 28.54 & 4.54 & 46.80 \\
\hline
\end{tabular}


Tabla 1. Información sobre el uso de las bibliotecas y los indicadores de desarrollo académico. Fuente: elaboración propia a partir de datos especificados en la sección de metodología y materiales (Rebiun, 2018; MECD, 2018).

Desde ahí, se calcularon los coeficientes de correlación de Pearson y Spearman de acuerdo a la normalidad de las variables y tal y como se explicó en la metodología, esto es, calculando el coeficiente de Spearman para las correlaciones entre los préstamos y la duración media de los estudios y las visitas y la duración media de los estudios. Los resultados de las correlaciones se presentan en la Tabla 2.

\begin{tabular}{|l|c|c|}
\hline \multicolumn{1}{|c|}{ Variables } & Préstamos per cápita & Visitas per cápita \\
\hline Tasa de rendimiento & $.457^{*}$ & .212 \\
\hline Tasa de éxito & $.485^{*}$ & .190 \\
\hline Tasa de eficiencia & $.523^{*}$ & .151 \\
\hline Tasa global de abandono & .045 & .106 \\
\hline Tasa de Graduación & $.483^{*}$ & .201 \\
\hline $\begin{array}{l}\text { Duración media de los estudios } \\
\text { (Spearman) }\end{array}$ & $-.474^{*}$ & -.197 \\
\hline
\end{tabular}

Tabla 2. Resultados para los coeficientes de correlación. Fuente: elaboración propia Nota: $n=41 ;{ }^{*} \mathrm{p} \leq 0,01$.

Como se puede ver, en el caso de los préstamos la relación resultó positiva y significativa con las variables de la tasa de rendimiento, tasa de éxito, tasa de eficiencia y la tasa de graduación. Por su lado, resultó negativa y significativa en el caso de los préstamos y la duración media de los estudios, correlación que fue calculada con el coeficiente de Spearman por la ausencia de normalidad de la última variable. Tan solo en el caso de la relación entre préstamos y la tasa global de abandono no se encontró una relación significativa. A su vez, los resultados de los coeficientes fueron cercanos a 0.5 en el caso de las relaciones positivas y a -0.5 en el de la negativa. Por ello puede decirse que la relación entre las variables resultaron positivas y moderadas en el primer caso y negativa y moderada en el segundo (Murray, 2015, p. 471), existiendo una relación lineal entre estas variables dada la existencia de significancia estadística.

Por su lado, los resultados de las relaciones entre las visitas y las variables del desarrollo académico resultaron no significativos en todos los casos y negativa solo en el de la duración media de los estudios.

Volviendo a las relaciones lineales significativas halladas para el caso de los préstamos y cinco de las variables del desarrollo académico, las relaciones positivas se aprecian claramente desde los gráficos de dispersión añadidos en el Gráfico 1. 

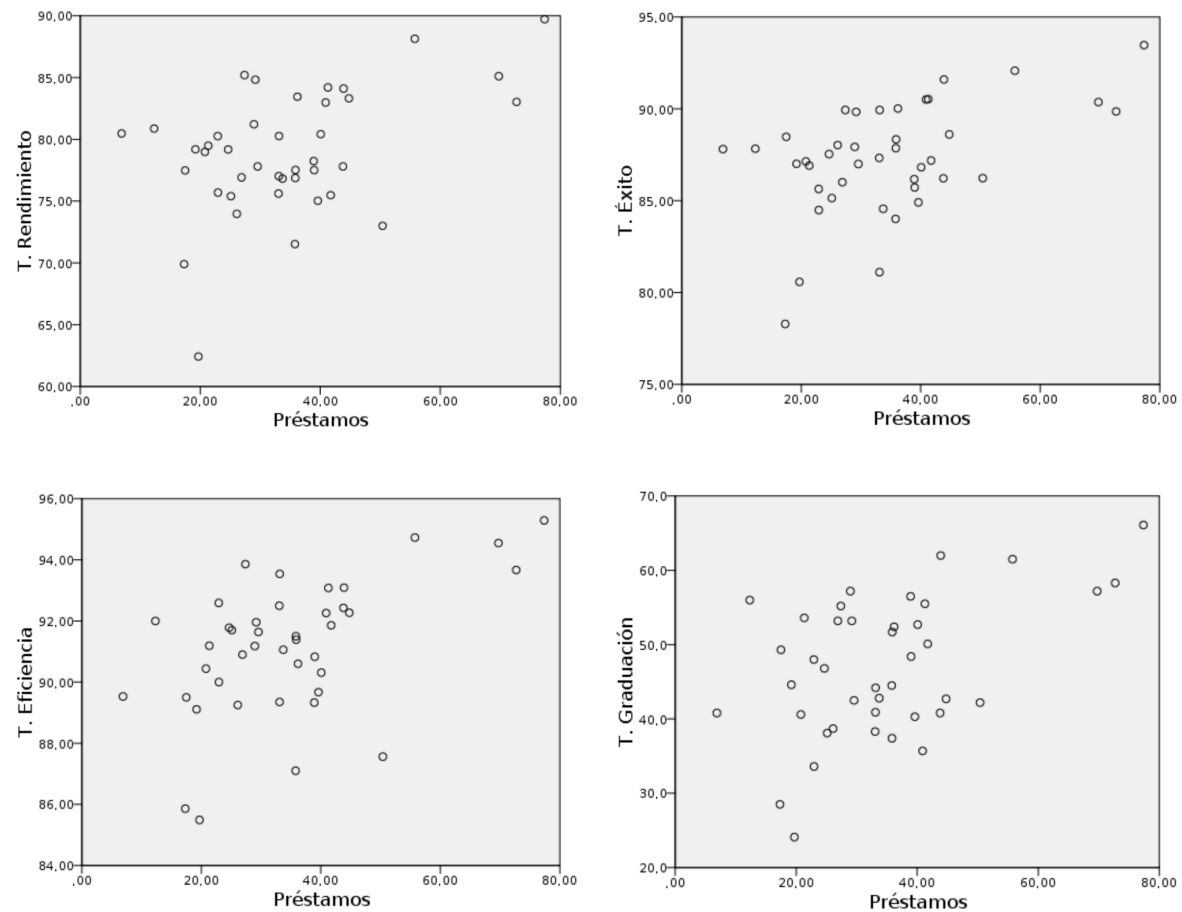

Gráfico 1. Diagramas de dispersión para las correlaciones halladas significativas positivas (variable préstamos per cápita y variables tasa de rendimiento, éxito, eficiencia y graduación). Fuente: elaboración propia.

Como puede verse, el eje horizontal representa en los 4 casos los préstamos per cápita, mientras que los ejes verticales las tasas de rendimiento, éxito, eficiencia y graduación respectivamente. De esta forma, se ve claramente la tendencia que marca la significancia estadística en la relación lineal positiva entre cada par de variables, de tal forma que en aquellas universidades (puntos en los gráficos) donde los préstamos en las bibliotecas han sido mayores, por lo general, las tasas anteriores también son mayores.

Por su lado, la representación gráfica para la relación lineal negativa entre los préstamos per cápita y la duración media de los estudios se presenta en el Gráfico 2. 


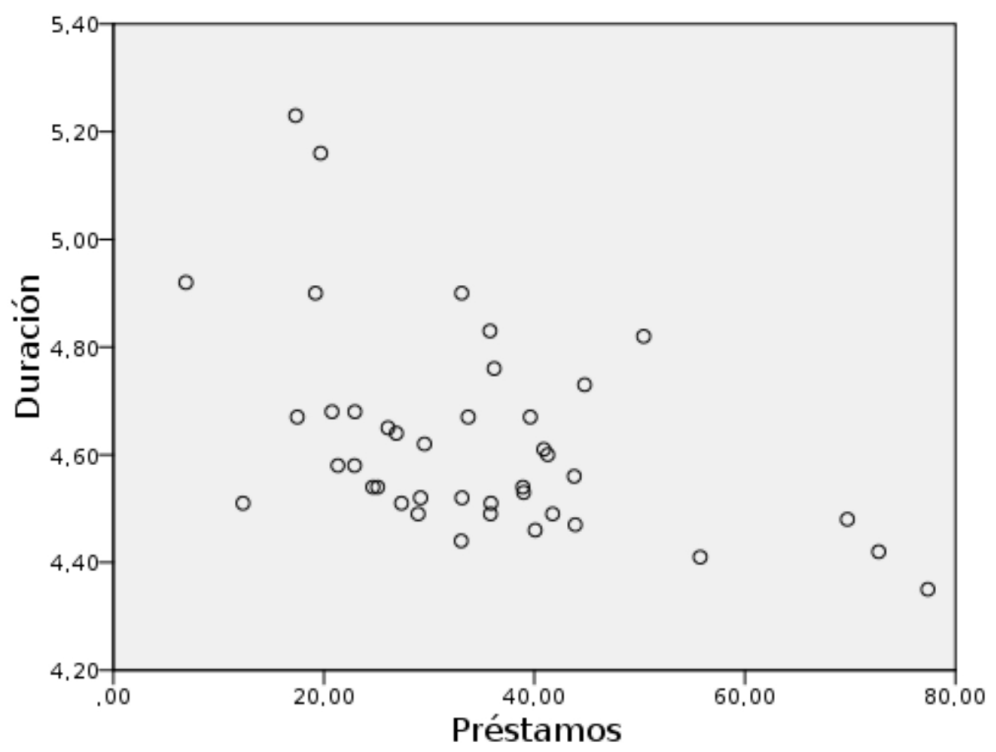

Gráfico 2. Diagrama de dispersión para la correlación hallada significativa negativa entre los préstamos per cápita y la duración media de los estudios. Fuente: elaboración propia.

En este caso, se aprecia también por lo general que en aquellas universidades donde los préstamos en las bibliotecas han sido mayores, la duración media en los estudios para estudiantes de grado es menor, tal y como indicó la significación estadística y el resultado del coeficiente de correlación o relación lineal negativa entre las variables.

Con todo, la interpretación de los resultados obtenidos permite aseverar, siempre con la precaución de no atribuir una relación de causalidad entre las variables, que en la muestra estudiada sucede que donde mayores son los préstamos per cápita en las bibliotecas públicas universitarias españolas, mayores son también los resultados para las tasas de rendimiento, de éxito, de eficiencia y de graduación, y menor es la duración media de los estudios en estudiantes de grado de dichas universidades. Por su lado, lo anterior no puede ser atribuido para el caso de las visitas per cápita a las bibliotecas.

\section{Discusión}

La pregunta de investigación en que se basa el presente estudio planteaba el estudio de la relación entre el uso de las bibliotecas universitarias públicas españolas y el desarrollo académico en estudiantes de grado. Considerando el 
uso de las bibliotecas desde los préstamos y las visitas, y el desarrollo académico desde las tasas de rendimiento, éxito, eficiencia, global de abandono, graduación, y la duración media de los estudios, la respuesta a la pregunta de investigación es que no se da relación entre las visitas y el desarrollo académico, pero sí entre los préstamos y dicho desarrollo.

En concreto, existe relación entre los préstamos y 5 de las variables del desarrollo académico: se da una relación lineal positiva moderada entre los préstamos y las tasas de rendimiento, éxito, eficiencia y graduación; y una relación lineal negativa moderada entre los préstamos y el caso de la duración media de los estudios.

Atendiendo a los trabajos que se mencionaron en la sección para la revisión de la literatura sobre la relación concreta entre éxito académico y uso de las bibliotecas, puede establecerse que los resultados obtenidos en el presente trabajo coinciden con la mayoría de los resultados de aquellos (Chohan et al., 2018; De Jager et al., 2017; Freitas-Hübner y Silveira-Andretta, 2016; MorićFilipović, 2012; Owusu-Acheaw, 2014; Wong y Webb, 2011; Yoon y Young, 2008). Por su lado, cabe decir que tan solo en uno de los estudios revisados se obtenía una limitada mejora en el éxito académico de los usuarios de bibliotecas al darse tan solo en una minoría de dichos usuarios (Alharbi y Middleton, 2011).

En ese sentido, si bien las variables en torno al uso de las bibliotecas de los estudios revisados no se reducen tan solo a los préstamos y las visitas, se reconoce en sus conclusiones que un mayor uso de las bibliotecas coincide con un mayor éxito académico en las muestras sobre las que trabajaban, algo que podría considerarse como hipótesis general de partida y que se cumple para el caso de los préstamos en el presente trabajo.

Desde ahí, puede decirse que una de las implicaciones más importantes del presente estudio es que el uso de las bibliotecas universitarias españolas entendido desde los préstamos puede ser un factor para un mayor desarrollo académico, siempre sin atribuir causalidad, o bien que el aumento de dicho desarrollo puede darse gracias a un mayor uso de las bibliotecas. Todo ello es, sin duda, un aporte de valor a dichas bibliotecas en el marco de la justificación de sus servicios a la institución a la que pertenecen, por cuanto pueden convertirse en un medio a tener en cuenta para mejorar el desarrollo académico de los estudiantes.

Por último en la discusión, en cuanto a la ausencia de relación entre visitas a la biblioteca y un mayor desarrollo económico podrían plantearse proyectos para conocer la valoración personal que los usuarios tienen de la dimensión de la biblioteca como espacio, tal y como se ha llevado a cabo en algunos estudios desarrollados en el campo de la evaluación de bibliotecas (López-Gijón, Ávila-Fernández, Pérez-Gálvez, y Herrera-Viedma, 2010, p. 255). $Y$ es que en un contexto donde los planes de estudio exigen el trabajo en grupo de los estudiantes, la adaptación de las bibliotecas a dicha exigencia ha de 
ser una prioridad en los modelos de gestión de las mismas.

\section{Conclusiones}

En este trabajo se ha estudiado la relación entre el uso de las bibliotecas de universidades públicas españolas y algunos indicadores de desarrollo académico para estudiantes de grado. La idea de partida se basaba en alcanzar un aporte de valor a las bibliotecas en caso de que un mayor uso de las mismas, fuese acompañado en la muestra estudiada de un mayor desarrollo académico. Así, en términos generales, se ha visto que efectivamente sucede lo anterior, si bien se puede matizar de acuerdo a las variables analizadas en cada caso.

Respecto de las visitas a las bibliotecas, no se obtuvo una relación lineal significativa con alguna de las variables del desarrollo académico, pero sí sucedió con el caso de los préstamos. Por lo que podemos decir que las bibliotecas de universidades públicas españolas pueden justificar sus servicios por el aporte y el valor que suponen dentro de la institución educativa a la que pertenecen. No obstante lo anterior, en los resultados se dejó claro de acuerdo a la metodología del estudio que un mayor uso de las bibliotecas no puede concebirse como la causa de un mayor desarrollo académico, pero sin duda y dada las implicaciones de esta investigación, puede ser considerado como un factor a su favor.

Las limitaciones del estudio vienen dadas en el sentido de que cubre un periodo de tiempo determinado (curso 2015-2016) y a unos estudiantes concretos (los de estudios de grado). En ese sentido, pueden diseñarse futuras investigaciones que amplíen la ventana temporal planteando estudios de series temporales sobre el tema. Por otro lado y tal y como se advirtió en la sección para la metodología y los materiales, los datos para los préstamos y las visitas a las bibliotecas no permiten diferenciar que préstamos y visitas han sido realizados y realizadas por según un tipo concreto de usuarios. Desde ahí, los resultados e implicaciones pueden ser criticados por dicho motivo, aunque una discusión mayor habría de considerar también que, por ejemplo, unos mayores préstamos y visitas del personal docente investigador a las bibliotecas podría significar también un aumento del desarrollo académico de los estudiantes de grado, y eso es algo que resultaría a favor de lo alcanzado en esta investigación.

Como otra posibilidad de investigación para el futuro, desde el campo de la biblioteconomía y la documentación se podría estudiar cómo conseguir que las visitas a las bibliotecas incidan como uno de los posibles factores para fomentar el desarrollo académico de los estudiantes. En ese sentido, atender a los planes de estudios concretos de cada grado para ver qué están requiriendo a los estudiantes (trabajos en grupo, nuevas fuentes de información, etc.), puede ser el punto de partida para adaptar los servicios de las bibliotecas a las necesidades de los usuarios en general y los estudiantes en particular. 
Con todo, gracias a este estudio se aporta valor a las bibliotecas universitarias generando a su vez temas e ideas que pueden ser desarrollados en un futuro para seguir aumentándolo y potenciándolo comparando lo que es con lo que podría llegar a ser, todo en virtud de la continua mejora de los servicios de las bibliotecas y el desarrollo académico.

\section{Bibliografía}

Alharbi, A. y Middleton, M. (2011). The relationship between academic library usage and educational performance in Kuwait. Library Management, 33(1/2), 86-94. Doi: https://doi.org/10.1108/01435121211203347.

Alonso-Arévalo, J. y Vázquez-Vázquez, M. (2018). La contribución de la biblioteca universitaria al logro de los planes y proyectos de la institución. Cuadernos de Documentación Multimedia, 29(1). Doi: https://doi.org/ 10.5209/CDMU.60033.

Boukacem-Zeghmouri, C., Bador, P., Lafouge, T. y Prost, H. (2016). Relationships between consumption, publication and impact in French universities in a value perspective: a bibliometric analysis. Scientometrics, 106(1), 263-280. Doi: https://doi.org/10.1007/s11192-015-1779-z.

Bustos-González, A. (2007). Bibliotecas universitarias: ¿sabemos medir sus resultados e impactos? El Profesional de la Información, 16(4), 281-286. Doi: https://doi.org/10.3145/epi.2007.jul.01.

Chohan, T.M., Bhatti, R. y Naeem, S. B. (2018). Prediction of Academic Performance of the University Students Through Their Use of Library Electronic Resources and Their Self-efficacy. Communications in Computer and Information Science, 810, 557-567. Doi: https://doi.org/10.1007/9783-319-74334-9_57.

De Jager, K., Nassimbeni, M., Daniels, W. y D'Angelo, A. (2017). The use of academic libraries in turbulent times: Student library behaviour and academic performance at the University of Cape Town. Performance Measurement and Metrics, 19(1), 40-52. Doi: https://doi.org/10.1108/PMM09-2017-0037.

Fernández, S. y Rubio, F. (2013). ¿El dinero importa? Relación entre el presupuesto de la biblioteca y la productividad investigadora de la Universitat Politècnica de València. Revista española de Documentación Científica, 36(4), e023. Doi: https://doi.org/10.3989/redc.2013.4.1043.

Freitas-Hübner, M. y Silveira-Andretta, P. (2016). A relação entre sucesso acadêmico e biblioteca universitária. Uma análise a partir dos empréstimos domiciliares em uma universidade brasileira. Información, cultura y sociedad: revista del Instituto de Investigaciones Bibliotecológicas, (34), 45-62. 
Herrera-Viedma, E. y López-Gijón, J. (2013). Libraries' Social Role in the Information Age. Science, 339(6126), 1382-1382. Doi: https://doi.org/ 10.1126/science.339.6126.1382-a.

Houghton, K., Foth, M. y Miller, E. (2013). The continuing relevance of the library as a third place for users and non-users of IT: the case of Canada Bay. The Australian Library Journal, 62(1), 27-39. Doi: https://doi.org/ 10.1080/00049670.2013.771764.

Kwak, S.-Y., y Yoo, S.-H. (2012). The public value of a national library: Results of a contingent valuation survey. Journal of Librarianship and Information Science, 44(4), 263-271. Doi: https://doi.org/ 10.1177/0961000612455820.

Lázaro-Rodríguez, P. y Vakkari, P. (2018). Modelizando el uso de las bibliotecas públicas través de sus características: estudio comparativo entre España y Finlandia para los préstamos y las visitas. Revista Española de Documentación Científica, 41(4): e216.

Lázaro-Rodríguez, P., López-Gijón, J., Alonso, S., Martínez-Sánchez, M.-Á. y Herrera-Viedma, E. (2018). Secaba-Rank, herramienta online para analizar y evaluar bibliotecas. El profesional de la información (EPI), 27(2), 278-288. Doi: https://doi.org/10.3145/epi.2018.mar.06.

Lázaro-Rodríguez, P., López-Gijón, J., Herrera-Viedma, E. (2018). Visibilidad de las bibliotecas públicas y la lectura en medios de comunicación españoles frente a otros hechos de la cultura y relación con su uso: medidas para su mayor promoción y difusión. BiD: textos universitaris de biblioteconomia $i$ documentació, (40). Doi: https://dx.doi.org/10.1344/BiD2018.40.7.

López-Gijón, J., Ávila-Fernández, B., Pérez-Gálvez, I.-J. y Herrera-Viedma, E. (2010). La calidad en las bibliotecas universitarias biomédicas según sus usuarios. El Profesional de la Información, 19(3), 255-259. Doi: https:// doi.org/10.3145/epi.2010.may.05.

MECD, (2018). Anuario de indicadores universitarios. Año 2017 - Ministerio de Educación, Cultura y Deporte. Recuperado de: https://tinyurl.com/ yaamq9jb.

Morić-Filipović, I. (2012). Evaluation of university library impact on students' academic success. Vjesnik bibliotekara Hrvatske, 55(1), 65-82.

Murray, A. (2015). Academic Libraries and High-Impact Practices for Student Retention: Library Deans' Perspectives. Portal: Libraries and the Academy, 15(3), 471-487. Doi: https://doi.org/10.1353/pla.2015.0027.

Murray, A., Ireland, A. y Hackathorn, J. (2016). The Value of Academic Libraries: Library Services as a Predictor of Student Retention. College \& Research Libraries, 77(5), 631-642. Doi: https://doi.org/10.5860/ crl.77.5.631.

Oppenheim, C. y Stuart, D. (2004). Is there a correlation between investment in an academic library and a higher education institution's ratings in the Re- 
search Assessment Exercise? Aslib Proceedings, 56(3), 156-165. Doi: https://doi.org/10.1108/00012530410699578.

Orduña-Malea, E. y Regazzi, J. J. (2014). U.S. academic libraries: understanding their web presence and their relationship with economic indicators. Scientometrics, 98(1), 315-336. Doi: https://doi.org/10.1007/s11192-0131001-0.

Owusu-Acheaw, M. (2014). Reading Habits Among Students and its Effect on Academic Performance: A Study of Students of Koforidua Polytechnic. Library Philosophy and Practice, 23(1130). Recuperado de: http://digitalcommons.unl.edu/libphilprac/1130.

Powell, R. R. (1992). Impact assessment of university libraries: A consideration of issues and research methodologies. Library and Information Science Research, 14(3), 245-257.

Rebiun. (2018). Estadísticas Rebiun - Red de Bibliotecas Universitarias Españolas. Recuperado de: http://rebiun.um.es/.

Soria, K. M., Nackerud, S. y Peterson, K. (2015). Socioeconomic Indicators Associated with First-year College Students' Use of Academic Libraries. The Journal of Academic Librarianship, 41(5), 636-643. Doi: https:// doi.org/10.1016/j.acalib.2015.06.011.

Wong, S. H. R. y Webb, T. D. (2011). Uncovering Meaningful Correlation between Student Academic Performance and Library Material Usage. College \& Research Libraries, 72(4), 361-370. Doi: https://doi.org/10.5860/ crl-129.

Yoon, Y. H. y Young, K. S. (2008). Correlation analysis between university research competitiveness and library's scholarly information in OECD nations and Korea. Scientometrics, 74(3), 345-360. Doi: https://doi.org/ 10.1007/s11192-007-1753-5. 